\title{
Review on prion diseases in animals with emphasis to Bovine Spongiform Encephalopathy
}

\author{
Mukund S. Londhe, Nand K. Mahajan, Rajender P. Gupta, Renu M. Londhe \\ Lala Lajpat Rai University of Veterinary \& Animal Sciences, Hisar - 125004, Haryana, India. \\ Corresponding author: Mukund S. Londhe, E-mail:drmukund.edu@gmail.com \\ Received: 04-02-2012, Accepted: 14-03-2012, Published Online: 06-05-2012 \\ doi: $10.5455 /$ vetworld.2012.443-448
}

\begin{abstract}
Prion diseases are known as Transmissible Spongiform Encephalopathies (TSE). These are degenerative brain disorders characterized by tiny microscopic holes that give the brain 'spongy' appearance. The causative agent is proteinaceous infective particle called prion. Prion diseases affect a variety of mammals including humans. The disease is transmitted by contaminated food or feed containing prion protein. In animals the diseases caused by prions are Scrapie, Bovine Spongiform Encephalopathy (BSE), Transmissible Mink Encephalopathy (TME), Chronic Wasting Disease (CWD), Feline Spongiform Encephalopathy (FSE) and exotic Engulate Encephalopathy (EUE). Currently the only reliable test is histo-pathological examination of tissues. Control measures are surveillance, culling sick animals and banning specified risk materials. In India no case of BSE has been reported so far but the disease warrants constant monitoring and surveillance if once introduced or imported would be a herculean task to eradicate it.
\end{abstract}

Keywords: Bovine Spongiform Encephalopathy, Brain disorders, Degenerative disease, Prion Disease, Proteinaceous.

Abbreviations: TSE - transmissible spongiform encephalopathies, BSE - bovine spongiform encephalopathy, $\operatorname{Pr} P c$ - normal prion protein, $\operatorname{Pr} P s c$ - abnormal prion protein.

\section{To cite this article:}

Londhe MS, Mahajan NK, Gupta RP, Londhe RM (2012) Review on prion diseases in animals with emphasis to Bovine Spongiform Encephalopathy, Vet World, 5(7):443-448, doi: 10.5455/vetworld.2012.443-448

\section{Introduction}

Prion diseases also known as transmissible spongiform encephalopathies (TSE) are a group of rare degenerative brain disorders characterized by tiny microscopic holes that give the brain 'spongy' appearance. The causative agent is a proteinaceous infective particle and from this the word Prion arises [1]. The Prion word was coined by Dr. Stanley B. Prusiner of the University of California, San Francisco in 1982 and he was awarded the Nobel Prize in 1997 for brilliant work on prion research.

These neurodegenerative diseases are incurable, with pathogenesis in the central nervous system, leading slowly but inexorably to death. A remarkable feature of this class of diseases is the extended period of time between exposure to the infectious agent and manifestation of clinical symptoms [2]. Prion diseases affect a variety of mammals including humans. In animals the diseases caused by prions are, scrapie in sheep and goats, bovine spongiform encephalopathy (BSE) in cattle (known as mad cow disease), transmissible mink encephalopathy (TME) in mink, chronic wasting disease (CWD) in white tailed deer, elk, mule deer and moose, feline spongiform encepha- lopathy in cats, exotic ungulate encephalopathy (EUE) in nyala and oryx.

In humans the disease caused by prions are Creutzfeldt-Jakob disease (CJD) and its varieties: iatrogenic Creutzfeldt-Jakob disease (iCJD), variant Creutzfeldt-Jakob disease (vCJD), familial Creutzfeldt-Jakob disease (fCJD), sporadic Creutzfeldt-Jakob disease (sCJD), GerstmannSträussler-Scheinker syndrome (GSS), Fatal familial insomnia (sFI) and Kuru [1].

\section{Etiology}

The causative agent is proteinaceous infective particle called prion [3]. Molecular weight of the protein particle is $30 \mathrm{kDa}$ and it does not contain nucleic acid. According to the prion theory, the agent of BSE is composed largely, but not entirely, of a selfreplicating protein, referred to as a prion. Another theory argues that the agent is virus-like and possesses nucleic acids which carry genetic information. Strong evidence collected over the past decade supports the prion theory, but the ability of the BSE agent to form multiple strains is more easily explained by a viruslike agent [4]. Main interesting and dreadful thing is, it does not produce any inflammatory or immune 
reaction in the host [5].

The normal prion protein is designated by the abbreviation PrPc. Prion-associated diseases develop when PrPc undergoes a structural change to form a structurally abnormal protein known as PrPsc [6]. The 'sc' designation is derived from the sheep disease scrapie. Once present, PrPsc is capable of inducing other normal PrPc molecules to undergo conformational change to the PrPsc form, resulting in the generation of extremely large number, of abnormal molecules [7]. PrPc is rich in $\alpha$-helical content and has little $\beta$-sheet structure, whereas PrPsc has less $\alpha$ helical content and is rich in $\beta$-sheet structure [8].

There is increasing evidence that there are different strains of BSE namely the typical BSE strain and two atypical strains ( $\mathrm{H}$ and $\mathrm{L}$ strains).

Typical BSE strain: The BSE strain responsible for most of the BSE cases in Canada is the same classic or typical strain linked to the outbreak in the United Kingdom. It is known to be preventable through elimination of BSE contaminated feed and has been causally linked to vCJD in humans. This typical strain has not yet been identified in any U.S.A. born cattle.

Atypical BSE strain: In July 2007, the UK Spongiform Encephalopathy Advisory Committee (SEAC) suggested that atypical BSE may be a distinct strain of prion disease. According to SEAC, the cases of atypical BSE may have risen spontaneously (although transmission through feed or the environment cannot be ruled out), unlike typical BSE. Recently reported French surveillance data support this theory that unlike typical BSE, atypical BSE appears to represent sporadic disease [9].

\section{Bovine spongiform encephalopathy}

Epidemiology: In Britain there was an increase in the use of animal protein derived from ruminants, as a cheaper source of protein for cattle feed in 1980s. This was necessitated following an increase in the world prices of protein supplement, such as fish meal and soya beans. The epidemiological evidence strongly indicates that disease was caused initially during the early 1980s, by feeding of rations containing meat and bone meal supplement to cattle, contaminated with the scrapie agent [10]. The prion protein can withstand high temperatures and does not get destroyed during the rendering procedure. Epidemiological studies indicate that this happened because of changes from high to low temperature rendering of sheep carcass material.

This changed process, introduced in 1980s appears to have been less efficient in inactivating the scrapie agent. When meat and bone meals processed in this manner were fed to cattle as part of their concentrate ration, the agent got introduced into the cattle [10]. Hence in July 1988, ruminant protein feed was banned in ruminants feed from 1986 to 2002. A total of 1,81,376 cases of BSE were confirmed in U.K and 4.4 million cattle slaughtered during the eradication programme [11]. The epidemic peaked in January 1993 with nearly 1000 new cases reported weekly. During the BSE epidemic in Britain, it was estimated that nearly one million cattle were infected with prions $[12,13]$. Almost two third of the dairy herds in the U.K. had at least one case of BSE [14]. Recently, in Portugal Annual incidence rate (Number of indigenous cases per million bovines) of BSE were 9.08 in 2009 and 6.83 in 2010 [15].

Currently, in Netherlands bovine spongiform encephalopathy events were found on August, 2010 and January 2011. After that in Switzerland also, outbreak of bovine spongiform encephalopathy was reported in April 2011 [16]. On February 18, 2011, the Canadian Food Inspection Agency (CFIA) confirmed a BSE case in a dairy cow born in Alberta in 2004 [9]. Since 1989, when the first BSE case was reported outside UK, a total of 1,88,556 cases have been reported from the number of different countries [4]. So far no case of BSE has been reported from India [17]. However, keeping in view the safety of Indian dairy herds and human health significance of BSE, the disease warrants constant monitoring and surveillance in India also; if once introduced or imported would be a herculean task to eradicate it [5]. By overlooking prion disaster in foreign countries it is now the duty of the government to educate the people for the same. So now government must to check each and every imported animals and protein supplements from other countries prior to import.

Transmission: While BSE is not contagious by direct contact, it is transmissible orally and parenterally [18]. Offal's of particular risk include brain, spinal cord, dorsal root ganglia and trigeminal ganglia [19]. There is no evidence of horizontal transmission of BSE among cattle [20]. There is no convincing evidence that maternal transmission plays a part in the transmission of BSE [21]. In short mainly, contaminated food or feed containing prion protein can transmit the disease.

Pathogenesis: One salient feature of the pathogenesis of BSE is reduced involvement of the lymphoreticular system (LRS) in comparison to that seen in other TSE diseases of other animals, including sheep scrapie [21]. After consumption of conta-minated feed containing prion protein, it passes through the 
intestinal mucosa without destructing by digestive juices or acids. It is not clear how prions pass through the intestinal mucosa. The incubation period is usually long of about 4-5 years [22]. It is believed that after oral uptake, prions may penetrate the intestinal mucosa through $\mathrm{M}$ cells and reach Payer's patches as well as the enteric nervous system [7]. Depending on the host other lymphoreticular tissue particularly spleen, but not lymph nodes is site of replication [5]. This is the case in sheep scrapie, but not BSE in cattle [7]. From the lymphoreticular system and possibly from other sites, prions proceed along the peripheral nervous system to finally reach the brain, either directly via the vagus nerve or via the spinal cord under involvement of the sympathetic nervous system [21].

1. Conversion of (PrP) to abnormal form: The normal protein PrP occurs naturally in the brains of animals and humans. Its function is not clearly understood, but in experimental models it appears to play a role in protecting cells and helping them respond to oxygen deficiency [21]. Conversion of normal animal protein PrP in the CNS occurs slowly and progressively to an abnormal form PrPsc by stimulation and this causes development of the disease [23]. Normally, prions are harmless, but when they are misshapen, they produce disease [24].

Prions cause no detectable immune or inflammatory response in the host [10]. This is because they occur naturally in animal and human body. Therefore they are not recognized as foreign and don't stimulate the immune system [25].

2. Progression of infected cell to death: The accumulation of abnormal prions in the brain causes neuronal cells to die and a type of protein called amyloid accumulates in plaques or flat areas and causes degeneration of brain tissue [5]. When the infected cells die, the defective prions are released into normal tissue and go on to infect more cells. Ultimately large clusters of cells die, leaving the brain filled with microscopic holes and a sponge like appearance under the microscope [26]. This is a prolonged slow process and symptoms of the disease may not appear for years [24].

Signs : BSE has a very long incubation period (about 4-5 years) and has an insidious onset [27]. Clinical signs include aggressive behaviour, gait and postural abnormalities which usually begin with apprehension, anxiety, fear, incoordination, difficulty in standing and extreme sensitivity to sound and touch i.e. hyperaesthesia [28]. These symptoms may progress for up to six months until the animal dies. Slaughter of the affected animals becomes necessary because of their unmanageable behaviour, traumatic damage as a result of repeated falling and prolonged recumbency [5].

Pathology: No gross post-mortem lesions were observed in BSE. The characteristic changes comprise in histopathologically were discrete ovoid and spherical vacuole or micro cavities in the neuropil. This spongiosis (i.e. neuropil vacuolation) is a predominant form of vacuolar change observed and is a feature of TSES [28]. The contents of vacuoles, both in the neuropil and in neurons remain unstained and clear, after histological staining for glycogen in paraffin and for lipids in fixed cryostat sections. A mild gliosis sometimes accompanies the degenerative changes [5].

\section{Chronic wasting disease (CWD)}

CWD is a transmissible spongiform encephalopathy of mule, deer, white-tailed deer, elk (wapiti) and moose. CWD has been found mainly in cervids (members of the deer family). As the only prion disease identified in free-ranging animals, CWD appears to be far more communicable than other forms of prion diseases. The disease was first recognized as a clinical "wasting" syndrome in 1967 in mule deer in a wildlife research faculty in northern Colorado, USA. It was identified as a TSE in 1978 and has spread to a dozen states. In captive populations, up to $90 \%$ of mule deer have been reported to be positive for prions [29]. The incidence of CWD in cervids living in the wild has been estimated to be as high as $15 \%$ [30].

The development of transgenic (Tg) mice expressing cervid $\operatorname{PrP}$ and thus susceptible to CWD, has enhanced detection of CWD and the estimation of prion titers $[31,32]$. Shedding of prions in the faeces, even in presymptomatic deer has been identified as a likely source of infection for these grazing animals [33]. CWD has been transmitted to cattle after intracerebral inoculation although the infection rate was low 4 of 13 animals [34]. This finding raised concerns that CWD prions might be transmitted to cattle grazing in contaminated pastures [35].

Most cases of CWD with clinical signs occur in adult animals. The disease is progressive and always fatal. The most obvious and consistent clinical sign of CWD is weight loss over time. Behavioural changes also occur in the majority of cases, including decreased interactions with other animals, listlessness, lowering of the head, blank facial expression and repetitive walking in set patterns. In elk, behavioural changes may include hyper excitability and nervousness. Affected animals continue to eat grain 
but may show decreased interest in hay. Excessive salivation and grinding of the teeth are also observed. Most deer show increased polydipsia and polyuria.

\section{Scrapie}

It is a fatal, degenerative disease that affects the nervous system of sheep and goat. Like others spongiform encephalopathies, scrapie is caused by a prion protein. Scrapie has been known since the 18th century (1732) and does not appear to be transmittable to humans. The name scrapie is derived from one of the symptoms of the condition, wherein affected animals will compulsively scrape off their fleece against rock, trees or fence.

The disease apparently causes an itching sensation in the animals. Other symptoms include excessive lipsmacking, strange gaits and convulsive collapse. The mechanism of transmission between animals and other aspects of the biology of the disease is only poorly understood and thus requires further research. The protein enters through the intestines which are gradually accumulated in the body, especially in nerve cells causing death of these cells. When the prions are absorbed through the intestines, they first appear in the lymph nodes, especially in payer's patches at the small intestine [35].

\section{Transmissible mink encephalopathy in mink}

Transmissible mink encephalopathy (TME) is a progressive and fatal neurodegenerative disease that affects ranched mink. Most of the adult animals on a ranch may be affected and once an animal exhibits clinical signs, death is inevitable. This disease is still poorly understood. It is very rare, with only a few outbreaks reported in the U.S.A. and other countries. Outbreaks seem to result from feeding contaminated food containing prions to mink; however, the origin of these prions is unknown. Recent evidence suggests they might be an unusual variant of the bovine spongiform encephalopathy (BSE) agent [36].

Several outbreaks of TME were reported in the United States of America between 1947 and 1985; no cases have been documented in the U.S.A. since that time. The incubation period is 6 to 12 months in ranched mink. The early clinical signs can be subtle, may include difficulty in eating, swallowing and changes in normal grooming behaviour. Affected mink often soil the nest or scatter faeces in the cage. Later, animals may become hyperexcitable and bite compulsively. Affected mink often carry their tails arched over their backs like squirrels. Once the clinical signs appear, TME is always progressive and fatal. Death usually occurs within 2-8 weeks [36].

\section{Feline Spongiform Encephalopathy (FSE)}

Feline spongiform encephalopathy (FSE) is a neurodegenerative disease, affects domesticated cats (housecats) and captive wild cats including cheetahs, pumas, ocelots, tigers, lions and Asian golden cats. Most cases have been seen in the United Kingdom. In addition, a few infected housecats have been found in Norway, Switzerland, Northern Ireland and Liechtenstein. Infected zoo cats have been reported from Australia, Ireland, France and Germany. Once the symptoms appear, this disease is invariably fatal. FSE was first reported in 1990 and was apparently transmitted to individual cats in BSE-contaminated food [37].

As the BSE epidemic has declined and controls have been placed on feeding high-risk bovine tissues to animals, FSE has become increasingly rare. However, this disease has a long incubation period and occasional cases continue to be reported in housecats and zoo animals. The lesion pattern emphasizes the spongy, change in the cerebrum, the corpus striatum, thalamus and cerebellar cortex [37].

\section{Diagnosis}

At present, there is no laboratory test available to diagnose BSE or TSE in the live animal. The routine laboratory diagnosis of TSE consists of histopathological examination of the brain and detection of the modified prion protein $\operatorname{PrP}$ (TSE) in brain tissue. These tests however, have the disadvantage of being laborious and time consuming so that results are available only after several days $[3,38]$.

Of the various diagnostic tests discussed, namely; histopatholoicalexamination, electron microscopic examination, immunohistochemistry, Western immunoblotting and ELISA but currently the only reliable test is histopathological examination of tissues after postmortem examination. A number of rapid tests are currently under validation [5].

\section{Control}

Control measures are surveillance, Culling sick animals, or banning specified risk materials or BSE infected meat for human food or for animal feed supplement. The most stringent control measures include a UK program that excludes all animals more than 30 months of age from the human food and animal feed supplies. The program appears to be highly effective.

In June 2000, the European Union Commission on Food Safety and Animal Welfare strengthened the European Union's BSE control measures by requiring 
all member states to remove specified risk materials from animal feed and human food chains as of October 1, 2000; such bans had already been instituted in most member states. Other control measures include banning the use of mechanically recovered meat from the vertebral column of cattle, sheep and goats for human food and BSE testing of all cattle more than 30 months of age destined for human consumption [39] is effective.

\section{Current research on prion}

Currently enormous research is going on prion protein and result is under progress. Presently suppression of prion propagation using poly-L-lysine by targeting plasminogen that stimulates prion protein conversion was carried out [40]. It was demonstrated that plasminogen stimulates $\operatorname{PrP}$ conversion as a cellular cofactor and anti-prion efficacy of plasminogen by using PLL. The results showed that PLL strongly inhibited PrPsc propagation in the cell-free, cell culture, and mouse models of prion disease. These results confirm the role of plasminogen in PrPsc propagation validates plasminogen as a therapeutic target to combat prion disease and suggests PLL as a potential anti-prion agent. Therefore, study represents a proof-of-concept that targeting plasminogen, a cofactor for PrP conversion, using PLL results in suppression of prion propagation, which represents a successful translation of our understanding on details of prion propagation into a potential therapeutic strategy for prion diseases.

\section{Conclusion}

As there is no reliable treatment or vaccines of prion diseases, so there is need to better understand the biology of prion diseases, to develop strategies to manage prion disease outbreaks and minimize the impacts and to apply learnings of prion diseases to the treatment. There is also necessity for further research to clearly understand how prions pass through the intestinal mucosa without being destroyed by digestive juices or acids. The pathogenesis of why prions cause no detectable immune or inflammatory responses in the host needs to be clarified and the pathogenesis of scrapie and transmissible mink encephalopathy (TME) needs be explained.

\section{Lessons learnt}

Animal husbandry plays key role in Indian economy so if BSE is introduced in cattle then there will be huge economic crisis and a great panic will spread all over the country. If BSE is once entered in
India then its impact can fall on farmers, consumers and society as it will result in great losses in animal husbandry and animal food industry. First of all it will be herculean task to stop spread of disease as there is no treatment so large number of animals has to slaughter and dairy, meat, hide, and animal product industry will suffer greatly. In India there is ban of cow slaughter in majority of states and this will create another problem due to religious aspects. By overlooking prion disaster in foreign countries it is now the duty of the government to educate the people for control and prevention. There should be constant surveillance of the prion diseases to avoid any kind of hazard. There must be a strict check on every imported animal and animal protein supplements from other countries prior to import.

\section{References}

1. Jubb, K.V.F., Keneddy, P.C., Palmer, N. (1993). Pathology of domestic animals, $4^{\text {th }}$ ed. Academic Press Inc., California, USA. 389-392.

2. Watts, J.C., Balachandran, A., Westaway, D. (2006). The expanding universe of prion diseases. PLoS. Pathog; 2(3): 26.

3. Neuendorf, E., Weber, A., Saalmueller, A., Schatzl, H., Reifenberg, K., Pffaff, E., Groschup, M.H.(2004). Glycosylation deficiency at either one of the two glycan attachment sites of cellular prion protein preserves susceptibility to bovine spongiform encephalopathy and scrapie infections. J. Biol. Chem; 279: 53306-16.

4. Bovine spongiform encephalopathy (2002). WHO media centre, www.who.int/mediacentre/factsheets /fs113/en/.

5. Vegad,J.L., Singh, R, Chauhan, R.S. (2008). Epidemiology and current concepts in the pathogenesis and diagnosis of bovine spongiform encephalopathy. Indian. J. Vet. Pathol; 32 (2): 125-134.

6. Port, T. (2007). Cause of mad cow disease: Suspected pathogens in bovine spongiform encephalopathy. Suitel0l.com.

7. Stokka, G.L., Boening (2000). Bovine spongiform encephalopathy. Kansas State University Agricultural Experiment Station and Cooperative Extensive Service, www. oznet.ksu.edu.

8. Pan, K.M., Baldwin, M., Nguyen, J., Gasset, M., Serban, A., Groth, D., Mehlhorn, I., Huang, Z., Fletterick, R.J., Cohen, F.E. (1993). Conversion of $\alpha$ helices into $\beta$-sheets features in the formation of the scrapie prion proteins. Proc. Natl. Acad. Sci; 90: 10962-10966.

9. Centers for disease control and prevention, CDC (2011).http://www.cdc.gov/ncidod/dvrd/bse/index.htm.

10. Vanopdenbosch, E., Roels, S. (2004). BSE: diagnosis and control, the consequences for human health and geographical BSE risk. Quebec/2004 
Canada.www.ivis.org.

11. Weissmann, C., Enari, M., Klohn, P.C., Rossi, D., Flechsig, E. (2002). Molecular biology of prions. Acta. Neurobiol. Exp; 62: 153-166.

12. Anderson, R.M., et al. (1996). Transmission dynamics and epidemiology of BSE in British cattle. Nature; 382: 779-788.

13. Nathanson, N., Wilesmith, J., Griot, C. (1997). Bovine spongiform encephalopathy (BSE): cause and consequences of a common source epidemic. Am. $J$. Epidemiol; 145: 959-969.

14. Kumar, R., Singh, R., Singh, K.P., Dwivedi, P., Chauhan, R.S. (2008) Background neurohistopathological lesions in Indian buffaloes (Bubalus bubalis). Indian J. Anim. Sci; 78:1211-22.

15. OIE (2011 B). Bovine spongiform encephalopathy Annual incidence rate of bovine spongiform encephalopathy (BSE) in OIE Member Countries that have reported cases, excluding the United Kingdom. http://www.oie.int/animal-health-in-the-world/bsespecific-data/annual-incidence-rate/.

16. OIE (2011 A). Bovine spongiform encephalopathy. BSE portal.http://www.oie.int/animal-health-in-theworld/bse-portal/\#oe_mainContent.

17. Singh, R., Kumar, R., Singh, P.P., Singh, K.P., Dwivedi, P., Chauhan, R.S. (2008). Neurohistopathological lesions in brains in Indian cattle. Indian J. Anim. Sci; 78(10): 1063-74.

18. Vegad, J.L. (2007). Mechanisms of prion-induced injury. A Textbook of Veterinary General Pathology, $2^{\text {nd }}$ ed. International book distributing company, Lucknow, India. 558-559.

19. Morley, R.S., Chen, S., Rheault, N. (2003). Assessment of the risk factors related to bovine spongiform encephalopathy. Rev. Sci. Tech. Off. Int. Epiz; 22: 157-178.

20. OIE (2000). Bovine spongiform encephalopathy. Animal diseases data. P. 1-7, http://www.oie.int/eng/ maladies/fiches/a_bll.htm.

21. Prince, M.J., Bailey, J.A., Barrowman, P.R., Bishop, K.J., Campbell, G.R. and Wood, J.M. (2003). Bovine spongiform encephalopathy. Rev. Sci. Tech. Off. Int. Epiz; 22: 37-60.

22. Bonnardiere, C.L. (2002). Bovine spongiform encephalopathy. Agricultura; 1:1-7.

23. Kupfer, L., Eiden, M., Buschmann, A., Groschup, M.H. (2007). Amino acid sequence and prion strain specific effects on the in vitro and in vivo convertibility of ovine/murine and bovine/murine prion protein chimeras. Biochim. Biophys. Acta; 1772: 704-13.

24. Cooley, W.A., et al. (2001). Evaluation of a rapid western immunoblotting procedure for the diagnosis of bovine spongiform encephalopathy (BSE) in the UK. $J$. Comp. Pathol; 125: 64-70.
25. Crosby, J.T. (2007). What causes mad cow disease, and how is this disease transmitted? about. com.

26. Nicholson, E.M., et al. (2008). Identification of a heritable polymorphism in bovine PRNP associated with genetic transmissible spongiform encephalopathy: evidence of heritable BSE. Open access. Plos. one. 3: 2912. Plos one/www.plosone.org.

27. Takashi, O. (2001). Recent progress in diagnosis for bovine spongiform encephalopathy, and studies in transgenic mice. Shokuniku. Ni. Kansuru. Josei. Kenkyu. Chosa. Seika. Hokokusho; 19: 43-48.

28. Prion (2005). Alberta Prion Research Institute. News \& Events. 1-6. htt://www.prioninstitute.ca/newsseptember2O_O5.aspx.

29. Williams, E.S. and Young, S. (1980). Chronic wasting disease of captive mule deer: A spongiform encephalopathy. J. Wildl. Dis; 16: 89-98.

30. Miller, M.W., et al. (2000). Epizootiology of chronic wasting disease in free-ranging cervids in Colorado and Wyoming. J. Wildl. Dis; 36: 676-690.

31. Browning, S.R., et al. (2004). Transmission of prions from mule deer and elk with chronic wasting disease to transgenic mice expressing cervid PrP. J. Virol; 78: 13345-13350.

32. Tamgüney, G., et al. (2006). Transmission of elk and deer prions to transgenic mice. J. Virol; 80: 9104-9114.

33. Tamgüney, G., et al. (2009). Asymptomatic deer excrete infectious prions in faeces. Nature; 461: 529-532.

34. Hamir, A.N., et al. (2001). Preliminary findings on the experimental transmission of chronic wasting disease agent of mule deer to cattle. J. Vet. Diagn. Invest; 13: 91-96.

35. Colby, D.W., Prusiner, S.B. (2011). Prions.Cold. Spring. Harb. Perspect. Biol;3(6):1-22.

36. The center for food security and public health (CFSPH) (2008). Transmissible Mink Encephalopathy. Iowa State University. 1-4. http://www.cfsph.iastate. edu/Factsheets/pdfs/Transmissible Mink Encephalopathy.pdf.

37. The center for food security and public health (CFSPH) (2007). Feline Spongiform Encephalopathy. Iowa State University. 1-5. www.cfsph.iastate.edu/ Factsheets/pdfs/feline spongiform encephalopathy.Pdf.

38. Keulen, V.L.J., et al. (2000). Diagnosis of bovine spongiform encephalopathy: a review. Vet. Q;(4):197-200.

39. Centers for disease control and prevention, CDC (2010). http://www.cdc.gov/ncidod/dvrd/bse/ prevention.htm.

40. Ryou, C., Titlow, W.B., Mays, C.E., Bae, Y. and Kim, S. (2011).The suppression of prion propagation using poly-L-lysine by targeting plasminogen that stimulates prion protein conversion. Biomaterials, 32(11):3141-9. 